\title{
Atrial Natriuretic Peptide (ANP)-Presence in Human Inferior Turbinate after Treatment Botulinum Toxin Type A Treatment
}

\author{
${ }^{1} \mathrm{~S}$. Incandela, ${ }^{1} \mathrm{C}$. Incandela, ${ }^{2} \mathrm{EV}$. Farina and ${ }^{3} \mathrm{~L}$. Lipari \\ ${ }^{1}$ Dipartimento di Biomedicina Sperimentale e Scienze Neurologiche Cliniche (BioNec). Sez. \\ Clinica Otorinolaringoiatrica, ${ }^{2}$ Anatomia Umana, ${ }^{3}$ Istologia ed Embriologia, \\ Universita di Palermo, 90127 Palermo, Argentina
}

\begin{abstract}
Studies demonstrated the ANP-presence in the mucosa of human turbinates, the study aimed to investigate about the ANP-presence of human turbinates of patients with rhinitis after treatment with the Botulinum Toxin-A (BTX-A) since no data are reported. Results show that in BTX-A treated patients ANP is present in the ciliated and basal cells of epithelium of tonaca mucosa and some glands; therefore ANP presence is more intense than in mucosa of untreated patients.
\end{abstract}

$\underline{\text { Key words: Human turbinates, patients, mucosa, ANP, basal cells, epithelium }}$

\section{INTRODUCTION}

Recently in the therapy of the symptoms in some rhinitis (such as vasomotor rhinitis or allergy) by intranasal administration the Botulinum Toxin type A (BTX-A) was used. Ozcan et al. (2006) found that the intranasal injection of BTX-A is a highly effective, safe and symptomatic treatment modality treatment with a long-lasting effect for patients with vasomotor rhinitis whose the pathophysiologic mechanisms are unknown. Experimental immunohistochemical studies were carried out in rats showed the VIP-presence (Wen et al., 2004) and in guinea pig showed apoptosis as a temporary phenomenon (Rohrbach et al., 2001). To date, previous studies demonstrated the Atrial Natriuretic Peptide (ANP)-presence in the mucosa of healthy patients in human inferior turbinate (Valentino et al., 2003) and in patients with hyperplasia but no study is reported about the presence of ANP both animals and human patients with the botulinum toxin A treatment. Aim of the study is to investigate about the ANP-presence in the tonaca mucosa of human turbinates of patients with rhinitis after treatment with the botulinum toxin-A since no data are reported. ANP is a peptide playing a vasodilative activity and it is present in the mucosa of normal patients and patients with allergy.

\section{MATERIALS AND METHODS}

The patient affected by allergic rhinitis was injected $20 \mathrm{U}$ of Botulinum Toxin A (BTX-A) into inferior turbinate, following the protocol (Ozcano et al., 2006).
Immunohistochemistry: For immunohistochemistry, the examples were fixed in Bouin's fluid, dehydrated in graded alcohols and embedded in paraffin. Seven micron thick sections were dewaxed in xylene passed through a graded series of alcohols and dehydrated in distilled water and were used to perform the ANP immunostaining, detected with avidin-biotin complex. Endogenous peroxidases were blocked by incubation with PBS containing 0.3\% hydrogen peroxide for $5 \mathrm{~min}$ at room temperature. Blockage of the unspecific binding sites was achieved with normal goat serum. The sections were treated with anti-ANP polyclonal antibody raised in rabbit (Chemicon) at serial dilution $1 / 500,1 / 600$ and $1 / 800$ in $0.05 \mathrm{M}$ Tris buffer, $\mathrm{pH} 7.2$, for $12 \mathrm{~h}$ at $4^{\circ} \mathrm{C}$, subsequently the section were washed in PBS ( 3 times for total $15 \mathrm{~min}$ ). The reaction was demonstrated with amino-c-ethyl-carbazole as substrate. Negative controls were sections treated with pre-immune serum or in which the primary antibody was omitted.

\section{RESULTS}

The observations on the turbinate of healthy patient show that the epithelium is some ANP-immunopositive cells, ciliated, basal cells and serous cells, the glands present immunonegative cells. The observations on turbinate of patients after the botulinum treatment evidence that the tonaca mucosa of turbinates present no alterations, the epithelium presents hyperplasia but it is well organized. In the tonaca propria, the glands present

Corresponding Author: Farina Elvira, Dipartimento Biomedicina Sperimentale e Neuroscienze Cliniche, Via del Vespro 129 , 90127 Palermo 


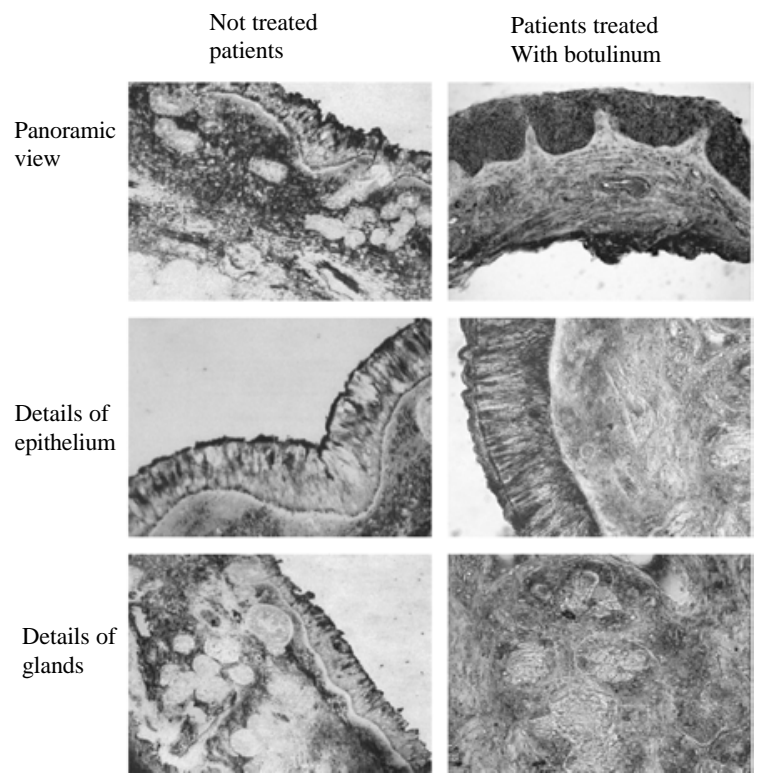

Fig. 1: ANP-immunopositive and immunonegative cells

some cells with picnotic nucleus. The ANPimmmunostaining shows detection of peptide in the epithelium with intense immunopositivity in the basal cells and in the ciliated cells and also in the superficial layer of the mucous. In the glands, some cells are ANPimmunopositive and some are ANP-immunonegative (Fig. 1).

\section{DISCUSSION}

The literature reports some experimental studies in rodents showed that in allergic ovalbumin sensitized rats the local BTX-A treatment reduced the clinical symptom as the rhinorrhea and that by the histological observations BTX treatment reduced edema, vasodilatation and inflammatory cell infiltration evidenced in allergic rats; furthermore and also evidenced that the density of VIP and SP-immunoreactive fibers decreased after the BTX-A treatment because of the reduction the neuron sensitivity of the mucosa (Wen et al., 2004, 2007). In guinea pig (Rohrbach et al., 2001) found that in the nasal mucosal tissue of the maxillary sinus turbinate the BTA-treatment after a short-term by TUNEL staining the degeneration of glands and ducts and apoptotic nuclei are observed but the glandular tissue is normal and thus the apoptosis is only temporary phenomenon. The study is carried out in human inferior turbinate and firstly demonstrates the ANP-presence in turbinate after the treatment with BTX-A. The results evidence that the treatment with botulinum determinates no change on the architecture and structure of the epithelium of tonaca mucosa of the turbinate and that the tonaca propria presents no edema. The glands also present no structural changes and the ANP-immunopositvity or ANP-immunonegativity of the cells it is only due to the functional moment. The BTX-A therapy seems to increase the presence of ANP.

\section{CONCLUSION}

The study concludes that the BTX-A treatment is of utility to reduce the clinical symptoms and evidences that it produces no alterations of the structure and the a more intense ANP-secretion both in the epithelium and in glands occurs.

\section{REFERENCES}

Ozcan, C., Y. Vayisoglu, O. Dogu and K. Gorur, 2006. The effect of intranasal injection of botulinum toxin $\mathrm{A}$ on the symptoms of vasomotor rhinitis. Am. J. Otolaryngol. Head Neck Med. Surg., 27: 314-318.

Rohrbach, S., A. Olthoff, R. Laskawi, B. Giefer and W. Gotz, 2001. Botulinum toxin type A induces apoptosis in nasal gland of guinea pig. Ann. Otol. Rhinol. Laryngol., 110: 1045-1050.

Valentino, B., D. Lipari, S. Restivo, R. Speciale, S. Gallina and E. Farina-Lipari, 2003. Localization of atrial natriuretic factor in the human inferior turbinates. An immunohistochemical study. Acta Otorhinolaryngol. Ital., 23: 94-97.

Wen, W.D., F. Yuan, Y.P. Hou and Y.F. Song, 2004. [Experimental studies for botulinum toxin type A on allergic rhinitis in the rat]. Zhonghua Er Bi Yan Hou Ke Za Zhi., 39: 97-101.

Wen, W.D., F. Yuan, J.L. Wang and Y.P. Hou, 2007. Botulinum toxin therapy in the ovalbumin-sensitized rat. Neuroimmunomodulation, 14: 78-83. 